\title{
RADIO TRANSIENTS FROM THE ACCRETION-INDUCED COLLAPSE OF WHITE DWARFS
}

\author{
Anthony L. Piro And S. R. Kulkarni \\ Cahill Center for Astrophysics, California Institute of Technology, Pasadena, CA 91125, USA; piro@ caltech.edu \\ Received 2012 November 2; accepted 2012 November 28; published 2012 December 13
}

\begin{abstract}
It has long been expected that in some scenarios when a white dwarf (WD) grows to the Chandrasekhar limit, it can undergo an accretion-induced collapse (AIC) to form a rapidly rotating neutron star. Nevertheless, the detection of such events has so far evaded discovery, likely because the optical, supernova-like emission is expected to be dim and short-lived. Here we propose a novel signature of AIC: a transient radio source lasting for a few months. Rapid rotation along with flux freezing and dynamo action can grow the WD's magnetic field to magnetar strengths during collapse. The spin-down of this newly born magnetar generates a pulsar wind nebula (PWN) within the $\sim 10^{-3}-10^{-1} M_{\odot}$ of ejecta surrounding it. Our calculations show that synchrotron emission from the PWN may be detectable in the radio, even if the magnetar has a rather modest magnetic field of $\sim 2 \times 10^{14} \mathrm{G}$ and an initial spin period of $\sim 10 \mathrm{~ms}$. An all-sky survey with a detection limit of $1 \mathrm{mJy}$ at $1.4 \mathrm{GHz}$ would see $\sim 4\left(f / 10^{-2}\right)$ above threshold at any given time, where $f$ is the ratio of the AIC rate to Type Ia supernova rate. A similar scenario may result from binary neutron stars if some mergers produce massive neutron stars rather than black holes. We conclude with a discussion of the detectability of these types of transient radio sources in an era of facilities with high mapping speeds.
\end{abstract}

Key words: stars: magnetic field - stars: neutron - stars: winds, outflows - white dwarfs

\section{INTRODUCTION}

As an accreting white dwarf (WD) grows toward the Chandrasekhar limit, a well-known potential outcome is ignition of its nuclear fuel, leading to a Type Ia supernova (SN Ia; Hillebrandt \& Niemeyer 2000). However, in some cases (e.g., mass transfer onto $\mathrm{O} / \mathrm{Ne} / \mathrm{Mg}$ WDs and $\mathrm{C} / \mathrm{O}$ WD mergers; Canal \& Schatzman 1976; Nomoto \& Kondo 1991) electron capture can rob the core of its degeneracy pressure support leading to formation of a neutron star (NS). This "accretion-induced collapse" (AIC) has been invoked to explain millisecond pulsars (e.g., Bhattacharya \& van den Heuvel 1991), subsets of gamma-ray bursts (e.g., Dar et al. 1992; Metzger et al. 2008b), magnetars (e.g., Usov 1992), and may be a source of $r$-process nucleosynthesis (Hartmann et al. 1985; Fryer et al. 1999).

Despite its potential importance, there has been no reported detection of an AIC event. To start with, the expected AIC rate is no more than $\approx 1 \%$ of that of $\mathrm{SNe}$ Ia (Yungelson \& Livio 1998). Next, relative to Type I and Type II SNe, the ejecta mass is expected to be small $\left(\lesssim 10^{-1} M_{\odot}\right)$, produce little ${ }^{56} \mathrm{Ni}$ $\left(\lesssim 10^{-2} M_{\odot}\right)$, and move at high velocity $(\approx 0.1 c)$. The resulting optical transient is thus considerably fainter than a typical SN (5 mag or more) and lasts $\sim 1$ day (Metzger et al. 2009b; Darbha et al. 2010).

Rapid rotation should accompany AIC due to the accretion of mass and angular momentum. Furthermore, a strong magnetic field may be amplified through flux freezing during collapse and via dynamo action (Duncan \& Thompson 1992). Therefore, a plausible outcome of AIC is creation of a quickly spinning magnetar (Usov 1992; King et al. 2001; Levan et al. 2006). The WD collapse unbinds material (Dessart et al. 2006), and the remnant disk loses mass via outflows driven by neutrino heating, turbulent viscosity, and recombination of free nuclei into helium (Lee \& Ramirez-Ruiz 2007; Metzger et al. 2008a, 2009a; Lee et al. 2009). This leads to $M_{\mathrm{ej}} \approx 10^{-3}-10^{-1} M_{\odot}$ of ejecta with velocity $v_{\mathrm{ej}} \approx 0.1 \mathrm{c}$. Such a configuration will also follow a double NS merger if the total binary mass is below the maximum NS mass.
Here we investigate the detectability of the pulsar wind nebula (PWN) powered by a newly formed magnetar following AIC. In Section 2 we describe a model for how the PWN expands into the surrounding ejecta. In Section 3 we estimate the radio spectrum and light curve, showing that the PWN is a transient radio source for a few months. We estimate the detection rate in Section 4 and discuss the detectability of such events with soon-to-be-commissioned high-speed radio mapping machines in Section 5.

\section{DYNAMICS OF THE PULSAR WIND NEBULA}

Following the implosion of the WD and disk outflows, the ejecta expands with velocity $v_{\mathrm{ej}} \approx 0.1 c$ and kinetic energy $E_{\mathrm{ej}} \approx M_{\mathrm{ej}} v_{\mathrm{ej}}^{2} / 2 \approx 10^{50} \mathrm{erg}$. The ejecta plows into the interstellar medium (ISM) with particle density $n_{0}$, which can vary greatly from $\sim 10^{-6} \mathrm{~cm}^{-3}$ for events outside of their host galaxy to $\sim 1 \mathrm{~cm}^{-3}$ in denser environments. At early times, the ejecta maintains constant velocity with radius $R_{\mathrm{ej}} \approx v_{\mathrm{ej}} t$. The forward shock is merely a distance $\approx(4 / 3)^{1 / 3} R_{\mathrm{ej}}$ ahead of $R_{\mathrm{ej}}$, and the reverse shock has barely developed behind it. The pressure behind the forward shock and down to the reverse shock is roughly constant and given by the strong shock limit $\approx(4 / 3) m_{p} n_{0} v_{\mathrm{ej}}^{2}$.

The magnetar injects energy in the form of magnetic fields and relativistic particles at a rate

$$
L(t)=L_{0} /(1+t / \tau)^{p},
$$

which powers a PWN with radius $R_{p}$. We assume dipole spindown ${ }^{1}$ and thus $p=2$. For magnetic moment $\mu$, initial spin frequency $\Omega_{0}=2 \pi / P_{0}$, and moment of inertia $I=0.35 M_{*} R_{*}^{2}$ (Lattimer \& Prakash 2001),

$$
L_{0}=\mu^{2} \Omega_{0}^{4} / 6 c^{3}=1.2 \times 10^{47} \mu_{33}^{2} P_{3}^{-4} \mathrm{erg} \mathrm{s}^{-1},
$$

\footnotetext{
1 When the magnetar is spinning $P_{0} \lesssim 3 \mathrm{~ms}$ and readily radiating neutrinos, this can give rise to a neutrino-driven, magneto-centrifugal wind (Thompson et al. 2004; Bucciantini et al. 2006), which could enhance the spin-down.
} 
and

$$
\tau=6 I c^{3} / \mu^{2} \Omega_{0}^{2}=4.8 \times 10^{4} \mu_{33}^{-2} P_{3}^{2} \mathrm{~s},
$$

where $\mu_{33}=\mu / 10^{33} \mathrm{G} \mathrm{cm}^{3}, P_{3}=P_{0} / 3 \mathrm{~ms}$, and we use $M_{*}=1.4 M_{\odot}$ and $R_{*}=12 \mathrm{~km}$.

The spin-down energy $E_{\text {spin }} \approx L_{0} \tau \approx 10^{52}$ erg exceeds $E_{\text {ej }}$, so quickly $E_{\text {spin }}$ dominates in setting the ejecta speed. Once the ejecta has swept up a mass comparable to its own, it would decelerate as it enters the Sedov-Taylor phase on a timescale (McKee \& Truelove 1995):

$$
\tau_{\mathrm{ST}}=0.5 E_{\mathrm{spin}}^{-1 / 2} M_{\mathrm{ej}}^{5 / 6}\left(m_{p} n_{0}\right)^{-1 / 3} \approx 2 E_{52}^{-1 / 2} M_{-2}^{5 / 6} n_{0}^{-1 / 2} \mathrm{yr},
$$

where $E_{52}=E_{\text {spin }} / 10^{52} \mathrm{erg}$ and $M_{-2}=M_{\mathrm{ej}} / 10^{-2} M_{\odot}$. But since $\tau_{\mathrm{ST}}$ is much longer than the times of interest for this work, the ejecta is always in an ejecta-dominated (or free-expansion) phase. Deeper inside, a wind termination shock forms at radius $R_{t}$, where the ram pressure of the pulsar wind equals the PWN pressure $P$ (Gaensler \& Slane 2006),

$$
R_{t} \approx(L / 4 \pi c P)^{1 / 2}
$$

where $R_{t} \ll R_{p}$. The general picture developed from galactic PWNe is that particles are accelerated at or near $R_{t}$. This seeds the PWN with relativistic electrons out to $R_{p}$.

A central issue is the content of the PWN. As a pulsar wind flows from the light cylinder, it is inferred to have a large magnetization ( $\sigma \sim 10^{4}$, where $\sigma$ is the ratio of Poynting flux to particle energy flux; Arons 2002). However, multiple lines of evidence, including the expansion velocities and high-energy modeling of PWNe, require that the magnetization decreases significantly $(\sigma \ll 1)$ by the time the wind reaches $R_{t}$. In the region where the radiation arises, $R_{t}<r<R_{p}$, we define $\eta_{e}$ and $\eta_{B}$ as the fraction of the magnetar luminosity that goes into electrons and magnetic fields, respectively, with typical values of $\eta_{e} \approx 0.999$ and $\eta_{B} \approx 10^{-3}$.

Following Reynolds \& Chevalier (1984), we assume the energy density of electrons and magnetic fields evolve independently and obey a relativistic equation of state. Thus,

$$
\frac{d}{d t}\left(4 \pi P_{e} R_{p}^{4}\right)=\left(\eta_{e} L-\Lambda\right) R_{p}
$$

where $\Lambda$ is the radiative loss rate, and

$$
\frac{d}{d t}\left(4 \pi P_{B} R_{p}^{4}\right)=\eta_{B} L R_{p},
$$

where $P_{B}=B^{2} / 8 \pi$. Note that in the adiabatic limit $\eta_{B} L \approx 0$, this predicts $P_{B} \propto R_{p}^{-4}$ and $B \propto R_{p}^{-2}$, as expected from flux freezing. Momentum conservation is given by

$$
M_{s} \frac{d^{2} R_{p}}{d t^{2}}=4 \pi R_{p}^{2}\left[P-\rho_{\mathrm{ej}}\left(v_{p}-\frac{R_{p}}{t}\right)^{2}\right],
$$

where $v_{p}=d R_{p} / d t$ and $P=P_{e}+P_{B}$ is the total pressure.

The PWN sweeps up ejecta and creates a shell with mass

$$
M_{s}=\left\{\begin{array}{ll}
M_{\mathrm{ej}}\left(R_{p} / v_{\mathrm{ej}} t\right)^{3}, & R_{p}<v_{\mathrm{ej}} t \\
M_{\mathrm{ej}}, & R_{p} \geqslant v_{\mathrm{ej}} t
\end{array} .\right.
$$

Once $R_{p} \approx v_{\mathrm{ej}} t$, the PWN reaches material that has been shockheated by its interaction with the ISM. Since we generally find $P \gg(4 / 3) m_{p} n_{0} v_{\mathrm{ej}}^{2}$, we do not expect the shock-heated pressure to have a large dynamical effect on the PWN. This is in contrast to PWNe growing within an SN remnant, where the large pressure behind the reverse shock (on a timescale $t \gg \tau_{\mathrm{ST}}$ ) can cause significant compression and magnetic field amplification (Reynolds \& Chevalier 1984).

Equations (6), (7), and (8) with $d R_{p} / d t=v_{p}$ provide four first-order differential equations for the four dependent variables $P_{e}, P_{B}, R_{p}$, and $v_{p}$, respectively. For simplicity, we calculate the PWN evolution using $\Lambda \approx 0$, so that Equations (6) and (7) are combined using $P=P_{e}+P_{B}$ and $\eta_{e}+\eta_{B}=1$. When $L$ is constant, the analytic result is (Chevalier 1977)

$$
R_{p} \approx 1.3 \times 10^{16} L_{47}^{1 / 5} E_{50}^{3 / 10} M_{-2}^{-1 / 2} t_{6}^{6 / 5} \mathrm{~cm},
$$

and

$$
B \approx 6 \eta_{B,-3}^{1 / 5} L_{47}^{1 / 5} E_{50}^{-9 / 20} M_{-2}^{3 / 4} t_{6}^{-13 / 10} \mathrm{G},
$$

where $L_{47}=L / 10^{47} \mathrm{erg} \mathrm{s}^{-1}, t_{6}=t / 10^{6} \mathrm{~s}$, and $\eta_{B,-3}=$ $\eta_{B} / 10^{-3}$. This gives some idea of the rough values expected, although for our detailed calculations, $R_{p}$ and $B$ deviate slightly from these scalings when $t \gtrsim \tau$.

Rayleigh-Taylor instabilities can act at $R_{p}$ due to the lowdensity PWN pushing up against high-density ejecta. The growth time for a large density contrast is $\tau_{\mathrm{RT}} \approx\left(g_{\text {eff }} k\right)^{-1 / 2}$, where $g_{\text {eff }}$ is the effective gravitational acceleration at the boundary and $k$ the wavenumber. For $R_{p}$ given by Equation (10), $g_{\text {eff }} \approx d^{2} R_{p} / d t^{2} \approx(6 / 25) R_{p} / t^{2}$ and the growth rate for $k \approx$ $n / R_{p}$ is $\tau_{\mathrm{RT}} \approx(25 / 6 n)^{1 / 2} t$. For sufficiently small wavelengths (large $n), \tau_{\mathrm{RT}} \lesssim t$ and instability results. This is not surprising since similar systems, like the Crab Nebula, have morphologies strongly impacted by instabilities. But, for the current analysis we do not include this complication.

\section{SYNCHROTRON RADIO EMISSION}

A power-law spectrum of relativistic electrons $n(E)=K E^{-s}$ is accelerated near the termination shock at $R_{t}$ and fills the PWN out to $R_{p}$, where $n(E)$ is in units of electrons $\mathrm{erg}^{-1} \mathrm{~cm}^{-3}$. For our fiducial models, we use $s=1.5$, motivated by Galactic PWNe (Bucciantini et al. 2011). The power-law parameters within the PWN can change via cooling and injection of new electrons (e.g., Gelfand et al. 2009). For the present work, we estimate the synchrotron spectrum at any time by fixing $P_{e}$ and $P_{B}$ from our dynamical calculations.

Our discussion of synchrotron emission largely follows the work of Pacholczyk (1970). Synchrotron emission is selfabsorbed below a frequency

$$
v_{\mathrm{SA}}=2 c_{1}\left(R_{p} c_{6}\right)^{2 /(s+4)} K^{2 /(s+4)}(B \sin \theta)^{(s+2) /(s+4)},
$$

where $c_{1}=6.27 \times 10^{18}$ in cgs units, $c_{6}$ depends on $s$ and can be found in Appendix 2 of Pacholczyk (1970), and $\theta$ is the pitch angle. Throughout we assume an average of $\sin \theta=(2 / 3)^{1 / 2}$. In the optically thick limit, the flux is

$$
F_{v}=\frac{\pi R_{p}^{2}}{D^{2}} \frac{c_{5}}{c_{6}}(B \sin \theta)^{-1 / 2}\left(\frac{v}{2 c_{1}}\right)^{5 / 2},
$$

where $D$ is the distance and $c_{5}$ is another constant. In the optically thin limit,

$$
F_{v}=\frac{4 \pi R_{p}^{3}}{3 D^{2}} c_{5} K(B \sin \theta)^{(s+1) / 2}\left(\frac{v}{2 c_{1}}\right)^{-(s-1) / 2},
$$



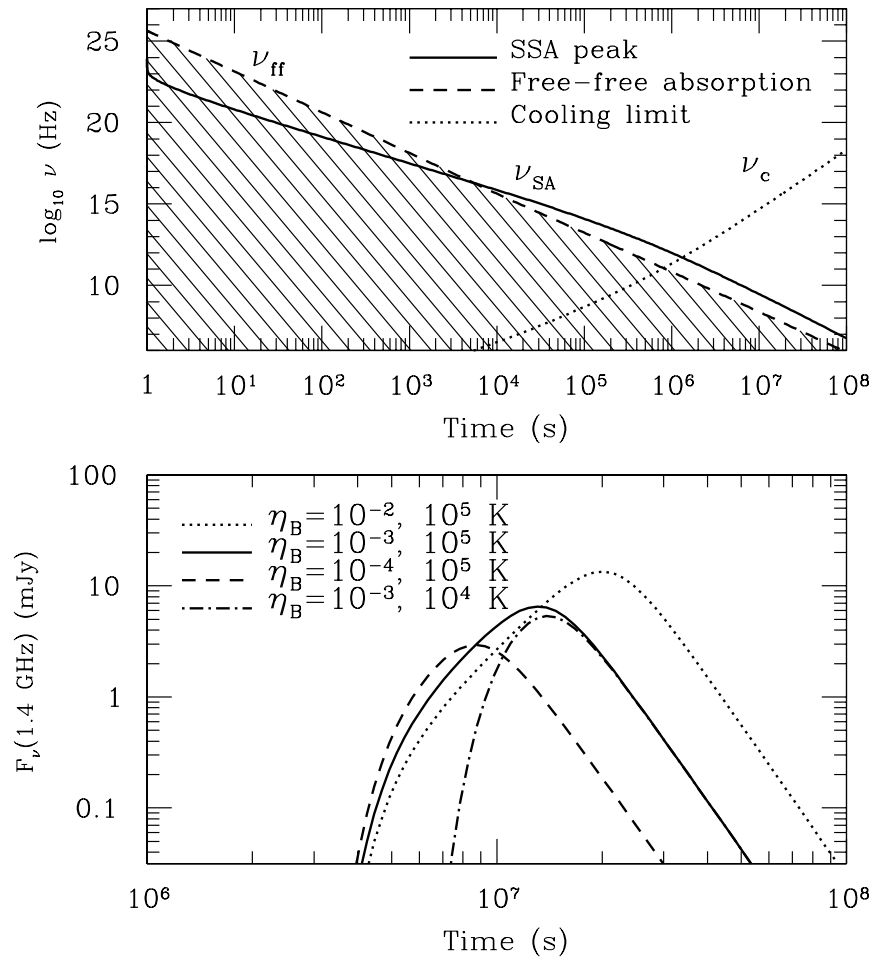

Figure 1. Top panel shows the evolution of the critical frequencies with time for $M_{\mathrm{ej}}=10^{-2} M_{\odot}, v_{\mathrm{ej}}=0.1 c, \mu=10^{33} \mathrm{G} \mathrm{cm}^{3}$, and $P_{0}=3 \mathrm{~ms}$. The electron spectrum has a power-law index $s=1.5$. The shaded region shows where free-free absorption suppresses the synchrotron spectrum for $T=10^{5} \mathrm{~K}$. This moves up for lower $T$ (see Equation (18)). The spectrum peaks at frequency $v_{\mathrm{SA}}$. The bottom panel shows the time-dependent flux at $v=1.4 \mathrm{GHz}$ for a distance $D=100 \mathrm{Mpc}$ over a range of parameters as labeled.

which assumes an emission filling factor of the order of unity. We use a simple interpolation between these two limits for the total emission spectrum $F_{v}$. The location of the spectrum's peak at $\nu_{\mathrm{SA}} \propto B^{(s+2) /(s+4)}$ shifts to lower frequencies as $B$ decreases during expansion (Equation (11)). The peak flux scales $\propto B^{(2 s+3) /(s+4)}$, and thus also decreases with time.

The synchrotron cooling time scales as $E /|\dot{E}| \propto v^{-1 / 2}$. Therefore, there is a $v_{c}$ above which synchrotron cooling beats adiabatic expansion (Reynolds \& Chevalier 1984):

$$
v_{c}=\frac{c_{1}}{c_{2}^{2} B^{3} \sin ^{3} \theta}\left(\frac{v_{p}}{R_{p}}\right)^{2},
$$

where $c_{2}=2.37 \times 10^{-3}$ in cgs units. Electrons that evolve adiabatically maintain their spectrum, while electrons that cool from synchrotron emission steepen, therefore

$$
n(E)= \begin{cases}K E^{-s}, & E<E_{c} \\ K E_{c} E^{-(s+1)}, & E \geqslant E_{c}\end{cases}
$$

where $E_{c}$ is the energy of electrons emitting a frequency $v_{c}$. The prefactor $K$ is set by the total energy density of electrons $U_{e}=3 P_{e}$, found by integrating

$$
U_{e}=\int_{E_{\min }}^{E_{\max }} n(E) E d E
$$

where $E_{\min }$ and $E_{\max }$ are the minimum and maximum energies of electron spectrum, respectively. For $s=1.5$ and the limit $E_{\max } \gg E_{c} \gg E_{\min }, U_{e} \approx 4 K E_{c}^{2-s}$. The energy density is roughly set by $E_{c}$ since the spectrum steepens for $E>E_{c}$.

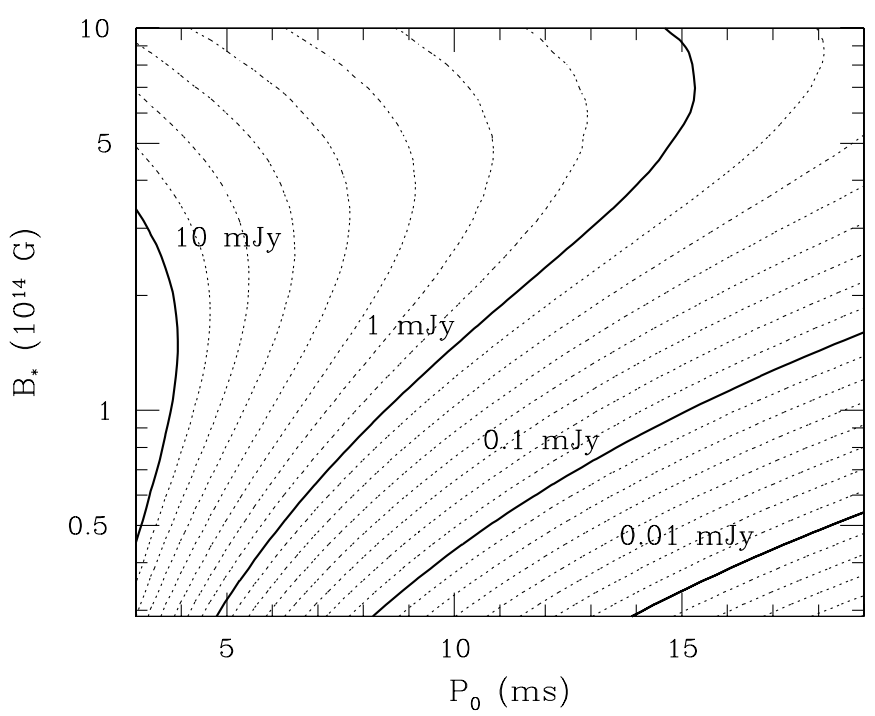

Figure 2. Peak flux at $1.4 \mathrm{GHz}$ at $100 \mathrm{Mpc}$ as a function of the magnetar field $B_{*}$ and its initial period $P_{0}$. The contours of constant peak flux are spaced logarithmically in units of mJy with the thick, solid curves marking the labeled contours. In all cases, we use $\eta_{B}=10^{-3}, T=10^{5} \mathrm{~K}$, and $s=1.5$.

We next consider potential free-free absorption. The absorption coefficient is (Rybicki \& Lightman 1979)

$$
\alpha_{\mathrm{ff}} \approx 1.9 \times 10^{-2} T^{-3 / 2} Z^{2} n_{e} n_{i} v^{-2} g_{\mathrm{ff}} \mathrm{cm}^{-1},
$$

for $h v \ll k_{\mathrm{B}} T$, where $T$ is the temperature, $Z$ is the average charge per ion, $n_{e}$ and $n_{i}$ are the electron and ion densities, respectively, $g_{\mathrm{ff}} \sim 1$ is the Gaunt factor, and all quantities are in cgs units. Just behind the forward shocks (see Section 2), the temperature is high $\left(\gg 10^{9} \mathrm{~K}\right)$, and free-free absorption is negligible. But, at the front edge of the ejecta, with density $\rho_{\mathrm{ej}}$ and temperature $T$, pressure continuity across the contact discontinuity requires $\rho_{\mathrm{ej}} k_{\mathrm{B}} T / m_{p} \sim n_{0} m_{p} v_{s}^{2}$. From this we estimate that $T \sim 10^{5}-10^{8} \mathrm{~K}$ at $t \sim 10^{6}-10^{7} \mathrm{~s}$. For $Z / A \approx 1 / 2$ (where $A$ is the average atomic number), we estimate $Z^{2} n_{e} n_{i} \sim$ $\left(\rho_{\mathrm{ej}} / m_{p}\right)^{2}$. The observed flux is thus $F_{v, \mathrm{obs}} \approx F_{v} e^{-\tau_{\mathrm{ff}}}$, where $\tau_{\mathrm{ff}} \approx \alpha_{\mathrm{ff}} \Delta R_{p}$ and $\Delta R_{p} \approx 0.1 R_{p}$ is the shell thickness.

In the top panel of Figure 1, we plot the evolution of the key frequencies $v_{\mathrm{SA}}$ (Equation (12)), $v_{c}$ (Equation (15)), and $v_{\text {ff }}$, where the latter is defined by $\tau_{\mathrm{ff}} \approx 1$. From this one can follow the spectrum peak (at $\nu_{\mathrm{SA}}$ ) and determine when it is detectable. For example, $10 \mathrm{GHz}$ emission peaks at $\approx 6 \times 10^{6} \mathrm{~s}$. In the bottom panel, we explore the light curves at $1.4 \mathrm{GHz}$ as we vary $T$ and $\eta_{B}$. Typical timescales are $\sim$ months with a peak of $\sim 3-10 \mathrm{mJy}$. Free-free absorption is mostly negligible unless $T \lesssim 10^{4} \mathrm{~K}$. The peak flux and time of peak are sensitive to $\eta_{B}$.

In Figure 2 we plot the peak flux at $1.4 \mathrm{GHz}$ for a range of dipole field strengths and initial spin periods. The general trend is that faster spins and larger fields result in a more luminous radio source. This reverses in the top left corner where $\tau$ is sufficiently short that $L(t)$ has decreased significantly by the time of peak. Over most of this parameter space, the magnetar winds are not expected to be strong enough to generate a collimated outflow (Bucciantini et al. 2012).

\section{DETECTION RATES}

AIC can result from both channels popularly discussed for SNe Ia (single degenerate and double degenerate; see Section 1). Thus it makes sense to normalize the AIC rate to that of the 
SN Ia rate. The Lick Observatory Supernova Search finds a rate of $(3.01 \pm 0.062) \times 10^{-5} \mathrm{Ia} \mathrm{Mpc}^{-3} \mathrm{yr}^{-1}(\mathrm{Li}$ et al. 2011),

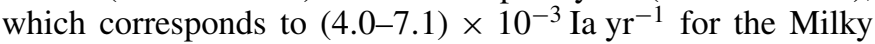
Way. Using population synthesis, Yungelson \& Livio (1998)

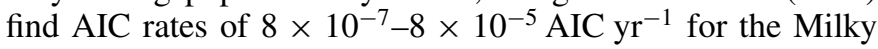
Way, depending on assumptions about the common-envelope phase and mass transfer. Their upper bound is similar to the constraint obtained from observed abundances of neutron-rich isotopes (Hartmann et al. 1985; Fryer et al. 1999).

For a typical peak $1.4 \mathrm{GHz}$ flux of $F_{p, 100} \sim 5 \mathrm{mJy}$ at a distance of $100 \mathrm{Mpc}$, and an AIC rate that is a fraction $f$ of the SN Ia rate, the detection rate of radio transients from AIC is

$$
\operatorname{Rate}\left(>F_{p, 100}\right) \approx 14\left(\frac{f}{10^{-2}}\right)\left(\frac{F_{p, 100}}{5 \mathrm{mJy}}\right)^{-3 / 2} \mathrm{yr}^{-1},
$$

where $f \sim 10^{-4}-10^{-2}$. For a $\sim 3$ month duration emitting above $\sim 1 \mathrm{mJy}$, we expect $\sim 4\left(f / 10^{-2}\right)$ AICs above threshold at a given time. In contrast, merely a few AICs are estimated to be detected as kilonovae per year (Metzger et al. 2009b), and this number could be much less if a lack of differential rotation during WD accretion (Piro 2008) inhibits disk formation upon collapse (Abdikamalov et al. 2010).

Similar radio emission is possible if some NS mergers produce a massive NS. Indeed, there is tantalizing evidence that the NSs in black widow system have masses well above $2 M_{\odot}$ (van Kerkwijk et al. 2011; Romani et al. 2012). If so, NS coalescence can contribute meaningfully to the magnetar channel. This would then provide an electromagnetic counterpart following months after the gravitational wave emission during coalescence $^{2}$ (Metzger \& Berger 2012; Nissanke et al. 2012). In fact, the distance at which the radio emission can be detected is similar to that probed by the next generation of "advanced" groundbased laser interferometers. The NS merger rate is comparable to or greater than the AIC rate. For example, Kim et al. (2005) estimate a Galactic rate of $\sim(0.1-3) \times 10^{-4} \mathrm{yr}^{-1}$. The detection frequency is thus similar to Equation (19) with $f=10^{-2}$, with the caveat that it may be much less if most NS mergers produce black holes.

\section{DETECTION AND IDENTIFICATION OF AIC EVENTS}

This is a timely topic to discuss given the renaissance that is now occurring in decimetric radio astronomy. Refurbished (Jansky Very Large Array (VLA)) and new facilities promise high mapping speeds by using small-diameter antennas to realize a given total area (e.g., MeerKAT), through the use of focal plane arrays instead of a single feed to gain massive multiplex advantage (APERTIF), or both (ASKAP).

We offer a plausible project aimed at detection of AICs that can be undertaken over the next few years. For a $7 \sigma$ detection $^{3}$ of a $1 \mathrm{mJy}$ point source, the Jansky VLA mapping speed in the $1.4 \mathrm{GHz}$ band (for a $500 \mathrm{MHz}$ bandwidth) is $\approx 86 \eta \mathrm{deg}^{2} \mathrm{hr}^{-1}$, where $\eta$ is the efficiency of time spent integrating on the sky (as opposed to slewing antennas or observing calibrator sources). For "on-the-fly" mapping, $\eta=0.9$ (S. Myers, private communication) and a $250 \mathrm{hr}$ allocation results in observing $\approx 19,000 \operatorname{deg}^{2}$ (comparable in area and depth to Faint Images

\footnotetext{
2 Also see the work of Nakar \& Piran (2011), which focuses on emission from the interaction of ejecta with the ISM.

3 In using a low threshold $(7 \sigma)$ we take advantage of the fact that galaxies in the local universe (say out to $100 \mathrm{Mpc}$ ) occupy a small solid angle ( $\sim 100 \mathrm{deg}^{2}$, excluding the nearest large galaxies), so chance coincidences are suppressed by three orders of magnitude (Kulkarni \& Kasliwal 2009).
}

of the Radio Sky at Twenty centimeters (FIRST); Becker et al. 1995). Another strategy would be to use the same allocation for several epochs focused on fields selected by their richness in nearby galaxies (as was done for the Palomar Transient Factory (PTF) key project "Investigation of Transients in the Local Universe"; Kasliwal 2011). A systematic optical mapping of the sky should be undertaken a week or more prior to the radio observations and continued for a week after (for instance, with PTF; Law et al. 2009; Rau et al. 2009).

The Jansky VLA radio survey would yield roughly two AICs. The signature of these events would be unique: they would be coincident with galaxies in the nearby Universe, would not be accompanied by any optical $\mathrm{SNe}$ of the sort that have detected so far, and would often occur outside of the nuclear regions of the host galaxy. For especially interesting radio detections, it may be worth conducting follow-up in the infrared to rule out the presence of an extinguished, radio-bright SN (although the steeper spectral index for the SNe may also be a useful discriminant; e.g., Chevalier 1998). The issue of associated $\mathrm{X}$-ray emission is less certain. It is possible that young magnetars shine in the X-rays, but if the ejecta is dominated by heavy elements then the classical X-ray emission is suppressed. A background active galactic nucleus or a foreground active star would be revealed by the distinctive spectra of the astrometric coincident quiescent source.

The future is quite bright. The soon-to-be-commissioned APERTIF (Verheijen et al. 2008) mapper could do the same survey $30 \%$ faster or alternatively redo the survey every few months. In the future, ASKAP and MeerKAT mappers could undertake the survey five times faster.

We thank Roger Chevalier, Peter Goldreich, Gregg Hallinan, Mansi Kasliwal, Keiichi Maeda, Brian Metzger, Christian Ott, E. Sterl Phinney, and Eliot Quataert. A.L.P. is supported through NSF grants AST-1212170, PHY-1151197, and PHY-1068881, NASA ATP grant NNX11AC37G, and the Sherman Fairchild Foundation. S.R.K.'s research is in part supported by NSF.

\section{REFERENCES}

Abdikamalov, E. B., Ott, C. D., Rezzolla, L., et al. 2010, PhRvD, 81, 044012 Arons, J. 2002, in ASP Conf. Ser. 271, Neutron Stars in Supernova Remnants, ed. P. O. Slane \& B. M. Gaensler (San Francisco, CA: ASP), 71

Becker, R. H., White, R. L., \& Helfand, D. J. 1995, ApJ, 450, 559

Bhattacharya, D., \& van den Huevel, E. P. J. 1991, PhR, 203, 1

Bucciantini, N., Arons, J., \& Amato, E. 2011, MNRAS, 410, 381

Bucciantini, N., Metzger, B. D., Thompson, T. A., \& Quataert, E. 2012, MNRAS, 419, 1537

Bucciantini, N., Thompson, T. A., Arons, J., Quataert, E., \& Del Zanna, L. 2006, MNRAS, 368, 1717

Canal, R., \& Schatzman, E. 1976, A\&A, 46, 229

Chevalier, R. A. 1977, in Astrophysics and Space Science Library, Vol. 66, Supernovae, ed. D. N. Schramm (Dordrecht: Reidel), 53

Chevalier, R. A. 1998, ApJ, 499, 810

Dar, A., Kozlovsky, B. Z., Nussinov, S., \& Ramaty, R. 1992, ApJ, 388, 164

Darbha, S., Metzger, B. D., Quataert, E., et al. 2010, MNRAS, 409, 846

Dessart, L., Burrows, A., Ott, C. D., et al. 2006, ApJ, 644, 1063

Duncan, R. C., \& Thompson, C. 1992, ApJL, 392, 9

Fryer, C., Benz, W., Herant, M., \& Colgate, S. A. 1999, ApJ, 516, 892

Gaensler, B. M., \& Slane, P. O. 2006, ARA\&A, 44, 17

Gelfand, J. D., Slane, P. O., \& Zhang, W. 2009, ApJ, 703, 2051

Hartmann, D., Woosley, S. E., \& El Eid, M. F. 1985, ApJ, 297, 837

Hillebrandt, W., \& Niemeyer, J. C. 2000, ARA\&A, 38, 191

Kasliwal, M. M. 2011, PhD thesis, California Institute of Technology

Kim, C., Kalogera, V., Lorimer, D. R., Ihm, M., \& Belczynski, K. 2005, in ASP Conf. Ser. 328, Binary Radio Pulsars, ed. F. A. Rasio \& I. H. Stairs (San Francisco, CA: ASP), 83

King, A. R., Pringle, J. E., \& Wickramasinghe, D. T. 2001, MNRAS, 320, L45 
Kulkarni, S., \& Kasliwal, M. M. 2009, in Proc. RIKEN Symposium, Astrophysics with All-Sky X-Ray Observations, ed. N. Kawai, T. Mihara, M. Kohama, \& M. Suzuki (Saitama: RIKEN), 312

Lattimer, J. M., \& Prakash, M. 2001, ApJ, 550, 426

Law, N., Kulkarni, S. R., Dekany, R. G., et al. 2009, PASP, 121, 1395

Lee, W. H., \& Ramirez-Ruiz, E. 2007, NJPh, 9, 17

Lee, W. H., Ramirez-Ruiz, E., \& López-Cámara, D. 2009, ApJL, 699, 93

Levan, A. J., Wynn, G. A., Chapman, R., et al. 2006, MNRAS, 368, L1

Li, W., Chornock, R., Leaman, J., et al. 2011, MNRAS, 412, 1473

McKee, C. F., \& Truelove, J. K. 1995, PhR, 256, 157

Metzger, B. D., \& Berger, E. 2012, ApJ, 746, 48

Metzger, B. D., Piro, A. L., \& Quataert, E. 2008a, MNRAS, 390, 781

Metzger, B. D., Piro, A. L., \& Quataert, E. 2009a, MNRAS, 396, 304

Metzger, B. D., Piro, A. L., \& Quataert, E. 2009b, MNRAS, 396, 1659

Metzger, B. D., Thompson, T. A., \& Quataert, E. 2008b, ApJ, 676, 1130

Nakar, E., \& Piran, T. 2011, Natur, 478, 82
Nissanke, S., Kasliwal, M., \& Georgieva, A. 2012, arXiv:1210.6362

Nomoto, K., \& Kondo, Y. 1991, ApJL, 367, 19

Pacholczyk, A. G. 1970, Radio Astrophysics (San Francisco, CA: Freeman)

Piro, A. L. 2008, ApJ, 679, 616

Rau, A., Kulkarni, S. R., Law, N. M., et al. 2009, PASP, 121, 1334

Reynolds, S. P., \& Chevalier, R. A. 1984, ApJ, 278, 630

Romani, R. W., Filippenko, A. V., Silverman, J. M., et al. 2012, ApJL, 760, 36

Rybicki, G. B., \& Lightman, A. P. 1979, Radiative Processes in Astrophysics (New York: Wiley)

Thompson, T. A., Chang, P., \& Quataert, E. 2004, ApJ, 611, 380

Usov, V. V. 1992, Natur, 357, 472

van Kerkwijk, M. H., Breton, R. P., \& Kulkarni, S. R. 2011, ApJ, 728, 95

Verheijen, M. A. W., Oosterloo, T. A., van Cappellen, W. A., et al. 2008, in AIP Conf. Ser. 1035, The Evolution of Galaxies through the Neutral Hydrogen Window, ed. R. Minchin \& E. Momjian (Melville, NY: AIP), 265

Yungelson, L., \& Livio, M. 1998, ApJ, 497, 168 